\title{
Impact of Symmetry on Anisotropic Magnetoresistance in Textured Ferromagnetic Thin Films
}

\author{
A. Philippi-Kobs $\odot,{ }^{1,2, *}$ A. Farhadi, ${ }^{2}$ L. Matheis, ${ }^{2}$ D. Lott, ${ }^{3}$ A. Chuvilin, ${ }^{4,5}$ and H. P. Oepen ${ }^{2}$ \\ ${ }^{1}$ Deutsches Elektronen-Synchrotron DESY, Notkestraße 85, 22607 Hamburg, Germany \\ ${ }^{2}$ Institut für Nanostruktur- und Festkörperphysik, Universität Hamburg, Luruper Chaussee 149, 22761 Hamburg, Germany \\ ${ }^{3}$ Institute for Materials Research, Helmholtz-Zentrum Geesthacht, Max-Planck-Straße 1, 21502 Geesthacht, Germany \\ ${ }^{4}$ Centro de Investigación Cooperativa nanoGUNE, Av. de Tolosa 76, E-20018 San Sebastian, Spain \\ ${ }^{5}$ IKERBASQUE, Basque Foundation for Science, Maria Diaz de Haro 3, E-480013 Bilbao, Spain
}

(Received 19 February 2019; published 25 September 2019)

\begin{abstract}
We report on the magnetoresistance of textured films consisting of $3 d$-ferromagnetic layers sandwiched by Pt. While the conventional $\cos ^{2} \varphi$ behavior of the anisotropic magnetoresistance (AMR) is found when the magnetization $\mathbf{M}$ is varied in the film plane, $\cos ^{2 n} \theta$ contributions $(2 n \leq 6)$ exist for rotating $\mathbf{M}$ in the plane perpendicular to the current. This finding is explained by the symmetry-adapted modeling of AMR of textured films demonstrating that the $\cos ^{2} \theta$ behavior cannot be used as a fingerprint for the presence of spin Hall magnetoresistance (SMR). Further, the interfacial MR contributions for Pt/Ni/Pt contradict the SMR behavior confirming the dominant role of AMR in all-metallic systems.
\end{abstract}

DOI: 10.1103/PhysRevLett.123.137201

Studies on magnetotransport in ferromagnetic materials have a long history starting with the experiments by Lord Kelvin in the middle of the 19th century $[1,2]$. Utilizing polycrystalline ferromagnetic wires the measurements revealed today's well-known anisotropic magnetoresistance (AMR) effect [3], which is a consequence of the spin-orbit interaction [4-6]. The AMR of single crystalline Fe and $\mathrm{Ni}$ was studied decades later [7-12] and a symmetry-adapted description for the observed dependence of resistivity on current and magnetization orientation with respect to the crystal axes was given by Döring [12]. The latter description is frequently used to successfully describe the AMR behavior of bulk crystals and single crystalline films [13-21]. Döring further demonstrated that the averaging over a random orientation of grains yields a $\cos ^{2} \phi$ variation of the resistivity $\rho$, with $\phi$ the angle between magnetization and currentdensity direction, which is commonly called AMR (herein referred to as conventional AMR) [12]. The conventional $\operatorname{AMR}\left(\Delta \rho_{\text {ip,bulk }}=\rho_{l, \text { bulk }}-\rho_{t, \text { bulk }}\right)$ is described as [using spherical coordinates, Fig. 1(a)] [12]

$$
\rho(\varphi, \theta)=\rho_{t, \text { bulk }}+\Delta \rho_{\text {ip,bulk }} \sin ^{2} \theta \cos ^{2} \varphi .
$$

In their famous review article McGuire and Potter derived from symmetry considerations the same angle dependence as Döring for the AMR of structurally isotropic polycrystalline films [22]. Nowadays, irrespective of any deviation from structural isotropy, the AMR of thin films is generally taken to be of the conventional form. The violation of isotropy, however, makes the a priori use of Eq. (1) to describe the AMR highly questionable. In the theoretical description of AMR, apart from a few recent exceptions [23,24], the impact of crystallinity on AMR is generally omitted and only the noncrystalline part [Eq. (1)] is modeled.

Textured films are prototypical for nonisotropic systems. In fact, additional AMR contributions were detected in such systems in polar rotational geometry $\left(\varphi=90^{\circ}\right)$ that solely depend on the polar angle, i.e., $\underbrace{\left(\rho_{p, \text { bulk }}-\rho_{t, \text { bulk }}\right)}_{\Delta \rho_{\text {op bulk }}} \cos ^{2} \theta$ [25-29]. The latter behavior is known as the geometrical size effect (GSE) although it was proven to be not a direct consequence of the finite size but caused by the film texture [29]. So far, it is not clear which underlying mechanism triggers the appearance of $\operatorname{GSE}\left(\Delta \rho_{\text {op,bulk }}\right)$ and how the relation to crystalline and conventional $\operatorname{AMR}\left(\Delta \rho_{\text {ip,bulk }}\right)$ is.

Some years ago, we demonstrated that the scattering at interfaces between ferromagnetic and paramagnetic layers made of heavy metals gives additional contributions to the AMR. In $\mathrm{Pt} / \mathrm{Co} / \mathrm{Pt}$ sandwiches contributions of this so-called anisotropic interface magnetoresistance (AIMR) were found in the conventional AMR geometry $\left(\Delta \rho_{\text {ip,interface }}\right)[30]$ as well as in polar rotational geometry $\left(\Delta \rho_{\text {op,interface }}\right)$ [31]. In general, the dependence of resistivity on magnetization orientation of textured thin films is then given by

$$
\begin{aligned}
\rho(\varphi, \theta)= & \rho_{t}+\left(\Delta \rho_{\text {ip,bulk }}+\Delta \rho_{\text {ip,interface }}\right) \sin ^{2} \theta \cos ^{2} \varphi \\
& +\left(\Delta \rho_{\text {op, bulk }}+\Delta \rho_{\text {op,interface }}\right) \cos ^{2} \theta .
\end{aligned}
$$

Signatures of $\Delta \rho_{\text {ip,interface }}$ and $\Delta \rho_{\text {op,interface }}$ were recently observed in various metallic layered structures [32-44]. 
The contributions of the interfaces cause a rather complex AMR behavior on thickness variation for films, sandwiches, and multilayers particularly in the range of ultrathin ferromagnetic layers $[45,46]$. Both AIMR terms were reproduced in a fully relativistic description of the magnetotransport in $\mathrm{Co} / \mathrm{Pt}(111)$ [23] where the spin-orbit interaction is inherently included; however, a plain explanation of the outcome was not given. In a semiclassical Boltzmann approach, both interface terms were derived from Bychkov-Rashba spin-orbit interaction at the interfaces [47]. A relation $\Delta \rho_{\text {op,interface }}=1.5 \Delta \rho_{\text {ip,interface }}$ was deduced which is in-line with the experimental outcome found for $\mathrm{Pt} / \mathrm{Co} / \mathrm{Pt}$ sandwiches [30].

Recently, magnetotransport in layered structures has again come into the focus of basic research triggered by the observation of phenomena related to the spin Hall effect $[48,49]$ like spin-orbit torque [50-52] and spin Hall magnetoresistance (SMR) [53,54]. The SMR effect present in insulating ferrimagnet-heavy-paramagnet systems leads to the following dependence of resistivity on magnetization orientation [53]:

$$
\begin{aligned}
\rho(\varphi, \theta) & =\rho_{t}+\Delta \rho_{\mathrm{ip}} \sin ^{2} \theta \cos ^{2} \varphi+\Delta \rho_{\mathrm{op}} \cos ^{2} \theta \quad \text { with } \\
\Delta \rho_{\mathrm{ip}} & =\Delta \rho_{\mathrm{op}} .
\end{aligned}
$$

Since the first pioneering experiment one branch of research deals with investigating the SMR in all-metallic multilayers; however, the outcome is not conclusive. As also pointed out by Zou et al. [45] the structural properties, particularly the crystallinity of the films, are mandatory for a profound interpretation of MR data; however, they are often disregarded or not considered [34,40-42,55-59] even in carefully performed studies $[60,61]$. Frequently, $\Delta \rho_{\text {op }}$ signatures are ad hoc interpreted to be a fingerprint of the SMR while not thoroughly proven whether crystalline or interfacial AMR effects exist [34,39-42,55-59,62]. The studies particularly lack an appropriate modeling of the electronic transport in all-metallic layered structures including the consideration of interface-scattering processes [39-42,55-61,63]. Properly correcting for the current shunt through paramagnetic layers provides the feasibility for the separation of interface and bulklike MR contributions [30], which is a necessary prerequisite to check for the validity of Eq. (3) $\left(\Delta \rho_{\text {ip }}=\Delta \rho_{\text {op }}\right)$ for the interfacial scattering processes.

In this Letter we report on the magnetoresistance of fcc (111)-textured $\operatorname{Pt}(5 \mathrm{~nm}) / \mathrm{Ni}\left(t_{\mathrm{Ni}}\right) / \mathrm{Pt}(3 \mathrm{~nm})$ sandwiches at room temperature for Ni thicknesses $2 \mathrm{~nm} \leq t_{\mathrm{Ni}} \leq 50 \mathrm{~nm}$. It is shown that the interfacial contributions violate the angular dependence of SMR [Eq. (3)]. Moreover, additional higher order contributions in $\cos ^{2 n} \theta$, with $2 n=4,6$, are found which are in contradiction to SMR theory. It is demonstrated that the complex behavior can be derived from crystalline AMR [12] when adapted to fcc (111) texture and uniaxial anisotropy of the interfaces providing a generic explanation for the existence of AIMR and GSE terms.

The preparation and compositional structure of the $\mathrm{Pt} / \mathrm{Ni} / \mathrm{Pt}$ films is similar to the one for $\mathrm{Pt} / \mathrm{Co} / \mathrm{Pt}[31,66]$. The structural characterization (Supplemental Material I [67]) particularly reveals that the grain size, crystallinity, and interfacial properties are independent on the $\mathrm{Ni}$ thickness enabling a thorough interpretation of the MR behavior. Importantly, the films are textured with a preferred [111] orientation of the crystal lattice of the grains along the film normal.

In order to determine the dependence of resistivity on magnetization orientation the samples were rotated in two planes with respect to the magnetic field direction, i.e., the rotation of magnetization in the film plane $[x, y$ plane, $\left.\theta=90^{\circ}\right]$ and in the plane perpendicular to the current direction $\left[y, z\right.$ plane, $\varphi= \pm 90^{\circ}$, Fig. $1(\mathrm{a})$; for details, see the Supplemental Material I [67]]. In the former case a $\cos ^{2} \varphi$ behavior is found for all Ni thicknesses [black dots, Fig. 1(b)] resembling the behavior of conventional AMR [Eq. (1)]. In polar rotational geometry $\rho(\theta)$ changes drastically its functional form on $\mathrm{Ni}$ thickness (red dots). While for the sandwich with the thinnest $\mathrm{Ni}$ layer $\left[t_{\mathrm{Ni}}=2 \mathrm{~nm}\right.$, upper graph in Fig. 1(b) $]$ an apparent $\cos ^{2} \theta$ behavior is observed with the global minima located at $\theta=\left(-90^{\circ}, 90^{\circ}\right)$ (transverse orientation of magnetization), the $t_{\mathrm{Ni}}=4 \mathrm{~nm}$ system (middle graph) behaves completely differently, showing two additional maxima and minima. Eventually, for the sandwich with $t_{\mathrm{Ni}}=15 \mathrm{~nm}$ (lower graph) the global minima are shifted to $\theta=\left(0^{\circ}, 180^{\circ}\right)$, i.e., the polar orientation of magnetization. In addition, small dips appear close to the maxima. The latter behavior is representative for $t_{\mathrm{Ni}} \geq 6 \mathrm{~nm}$ as can be seen from Fig. 1(d), where the $\rho(\theta)$ curves for all Ni thicknesses are displayed in a polar plot.

A Fourier analysis reveals that orders of $\cos ^{2 n} \theta$ with $2 n \leq 6$ have to be considered to properly describe the $\rho(\theta)$ curves [Fig. 1(c)]. The rotational curves for all Ni thicknesses were fitted by

$\rho(\varphi)=\rho_{t}+\Delta \rho_{\mathrm{ip}} \cos ^{2} \varphi$ and $\rho(\theta)=\rho_{t}+\sum_{n=1}^{3} \Delta \rho_{\mathrm{op}}^{(2 n)} \cos ^{2 n} \theta$,

respectively, and the amplitudes $\Delta \rho_{\mathrm{ip}}$ and $\Delta \rho_{\mathrm{op}}^{(2 n)}$ were extracted. The corresponding MR ratios $\Delta \rho_{\text {ip }} / \rho$ and $\Delta \rho_{\mathrm{op}}^{(2 n)} / \rho$ are plotted vs Ni thickness in Fig. 2. The thickness dependence of $\Delta \rho_{\text {ip }} / \rho$ [Fig. 2(a)] is very similar to the $\Delta \rho_{\text {ip }} / \rho\left(t_{\mathrm{Co}}\right)$ behavior observed for $\mathrm{Pt} / \mathrm{Co} / \mathrm{Pt}[30,31]$. Again, the drop at small thicknesses is predominantly caused by current shunting through the Pt layers [31]. The $\Delta \rho_{\mathrm{op}}^{(2 n)} / \rho\left(t_{\mathrm{Ni}}\right)$ behaviors shown in Fig. 2(b) demonstrate that the second and fourth orders are dominant; hence their interplay dictates the $\rho(\theta)$ dependence, i.e., in 


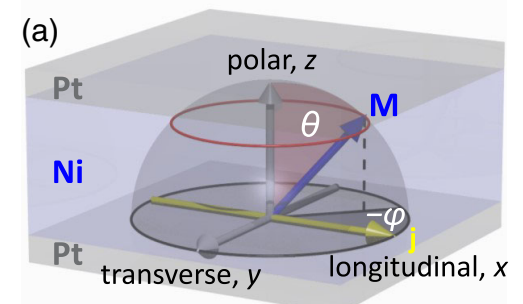

(b)

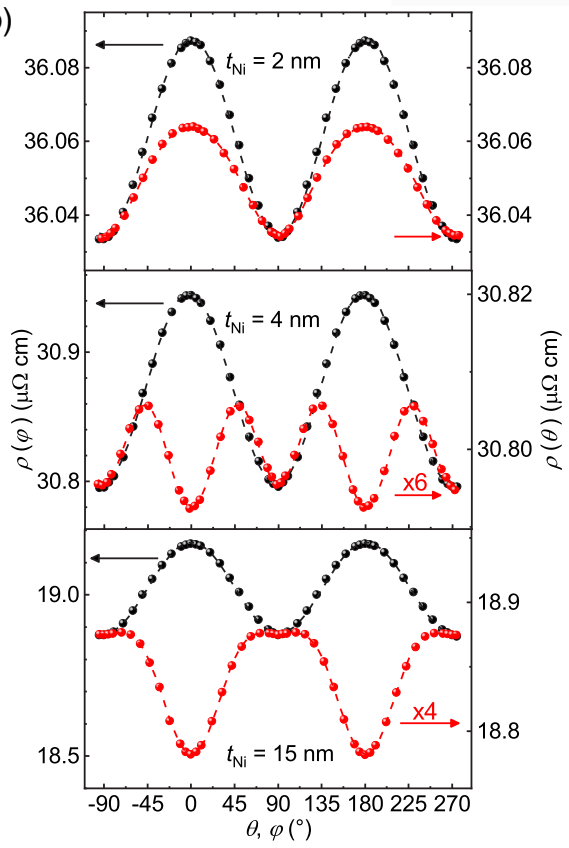

(c)

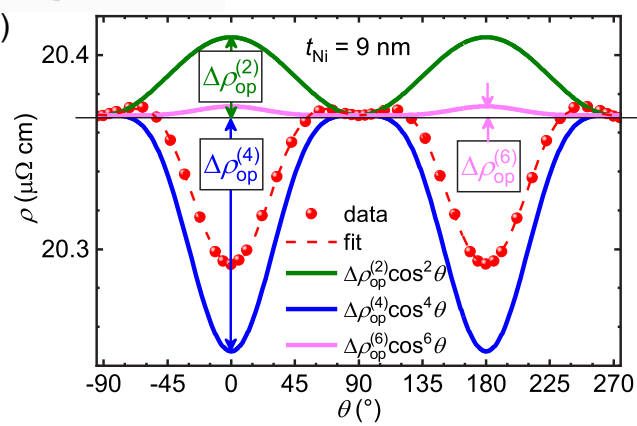

(d)

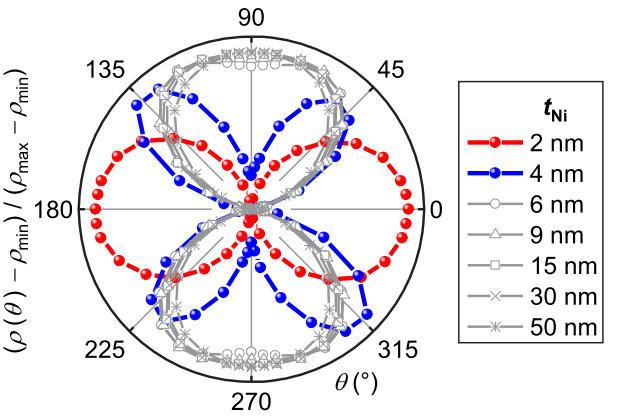

FIG. 1. MR behavior of Ni/Pt layered structures. (a) Sketch of a Pt/Ni/Pt sandwich and the experiment-related coordinate system. (b) Resistivity $\rho$ vs orientation of magnetization for three different Ni thicknesses [ $t_{\mathrm{Ni}}=2 \mathrm{~nm}$ (top), $4 \mathrm{~nm}$ (middle), $15 \mathrm{~nm}$ (bottom)]. The rotation of magnetization $\mathbf{M}$ is performed in the film plane $\left[\theta=90^{\circ}\right.$, black dots] and in the plane perpendicular to the current $\mathbf{j}$ $\left[\varphi=90^{\circ}\right.$ for $\theta=\left(0^{\circ}, 180^{\circ}\right)$ and $\varphi=-90^{\circ}$ for $\theta=\left(-90^{\circ}, 0^{\circ}\right)$ and $\left(180^{\circ}, 270^{\circ}\right)$, red dots $] . \rho\left(\varphi, \theta=90^{\circ}\right)$ follows the conventional AMR behavior for all $t_{\mathrm{Ni}}$ ( $\cos ^{2} \varphi$ fits, black dashed lines) while the functional dependence of $\rho\left(\varphi= \pm 90^{\circ}, \theta\right)$ varies with $t_{\mathrm{Ni}}$. The red dashed lines are fits to the data using a $\cos ^{2 n} \theta$-series expansion [Eq. (4)]. (c) Individual $\cos ^{2 n} \theta$ contributions to $\rho\left(\varphi= \pm 90^{\circ}, \theta\right)$ depicted for $t_{\mathrm{Ni}}=9 \mathrm{~nm}$. (d) Polar plot of $\rho\left(\varphi= \pm 90^{\circ}, \theta\right)$ for all samples.

particular, the location of the resistivity minima and maxima. The sixth order is very small and therefore omitted in the following discussion [73]. The thickness dependence of $\Delta \rho_{\mathrm{op}}^{(2)} / \rho$ is similar to the $\Delta \rho_{\mathrm{op}}^{(2)} / \rho\left(t_{\mathrm{Co}}\right)$ behavior found for $\mathrm{Pt} / \mathrm{Co} / \mathrm{Pt}$ [31]. Hence, the $1 / t_{\mathrm{Ni}}$ dependence indicates the presence of an interface contribution while the behavior at large $t_{\mathrm{Ni}}$ reflects the bulk contribution (GSE) which has an opposite (negative) sign. In contrast to $\mathrm{Pt} / \mathrm{Co} / \mathrm{Pt}$, however, a remarkably strong fourth-order contribution exists for $\mathrm{Pt} / \mathrm{Ni} / \mathrm{Pt}$ that dominates for $t_{\mathrm{Ni}} \geq 6 \mathrm{~nm}$. Qualitatively, $\Delta \rho_{\mathrm{op}}^{(4)} / \rho$ exhibits a similar $t_{\mathrm{Ni}}$ dependence as $\Delta \rho_{\mathrm{op}}^{(2)} / \rho$ apart from the fact that $\Delta \rho_{\mathrm{op}}^{(4)} / \rho$ is negative throughout the whole thickness range. The Ni-thickness dependence of the MR terms is discussed further below when presenting the results of the analysis that provides a careful separation of interface and bulk-MR contributions.

To summarize, besides the complex MR behavior described by Eq. (2) higher order contributions of $\cos ^{2 n} \theta$,

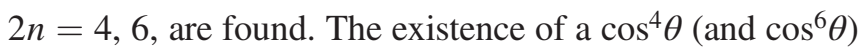
term has not been reported for thin polycrystalline films before. Importantly, higher orders are incompatible with SMR [74] and Rashba-based AMR effects [47] which strictly follow a $\cos ^{2}$ behavior. Higher orders in the AMR, however, were frequently found for single-crystalline systems $[12,13,21,75]$ satisfying the symmetry-adapted description [12]. The latter fact triggered the idea to derive the AMR of fcc (111)-textured films under consideration of the underlying symmetry to check for the compatibility with the experimental outcome.

The longitudinal resistivity can be described by a series expansion with respect to current direction and magnetization orientation, both of which have to be expressed in the reference frame of the crystal $[12,22,76]$. As a starting point the series expansion for fcc single crystals is used [12,77]. The AMR of fcc (111)-textured films is obtained by averaging the single-crystal behavior over all 


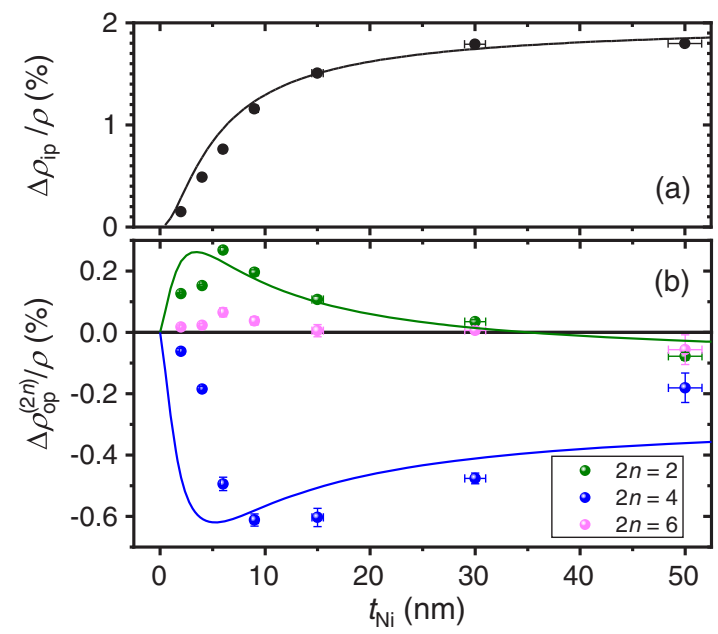

FIG. 2. MR ratios (a) $\Delta \rho_{\text {ip }} / \rho$ and (b) $\Delta \rho_{\mathrm{op}}^{(2 n)} / \rho$ as a function of $\mathrm{Ni}$ thickness $t_{\mathrm{Ni}}$. The solid lines are model curves (see details in the Supplemental Material III [67]). The relatively strong difference between experimental data and model curves for $t_{\mathrm{Ni}} \leq 6 \mathrm{~nm}$ indicates the presence of magnetic dead layer formation at the $\mathrm{Ni} / \mathrm{Pt}$ interfaces.

in-plane orientations to account for the random in-plane orientation of the individual grains (derivation, Supplemental Material II [67]). The general result for the bulk contribution by using spherical coordinates is

$$
\frac{\Delta \rho(\varphi, \theta)}{\rho}=A+B_{\mathrm{ip}} \sin ^{2} \theta \cos ^{2} \varphi+B_{\mathrm{op}} \cos ^{2} \theta+C_{\mathrm{op}} \cos ^{4} \theta .
$$

Obviously, the experimental results [Eq. (4)] are in accordance with Eq. (5) [79]. In particular, Eq. (5) proves that the assumption of a conventional AMR behavior [Eq. (1)] is justified for the in-plane rotation of the magnetization $\left(\theta=90^{\circ}\right)$ demonstrating that the expansion is terminated with the second order in $\cos ^{2 n} \varphi$. The second characteristic of conventional AMR, i.e., a constant resistivity when rotating the magnetization in the $y, z$ plane $\left(\varphi=90^{\circ}\right)$, is not fulfilled. Quite to the contrary, besides a second-order also a fourth-order contribution in $\cos ^{2 n} \theta$ can generally be expected. While the second-order term has been frequently found, i.e., the GSE [25,29,31], higher orders have not been reported for textured films so far. From our treatment using strict symmetry considerations it becomes evident that the GSE is not due to texture-induced $s$ - $d$ scattering as speculated in Ref. [29] but a mere consequence of the crystallinity and hence anisotropic orientation of the grains.

The general result [Eq. (5)] leads immediately to the question for the MR behavior in other layered film systems. For instance, for $\mathrm{Pt} / \mathrm{Co} / \mathrm{Pt}$ sandwiches second-order contributions were reported frequently $[31,37,59,60]$. Hence,

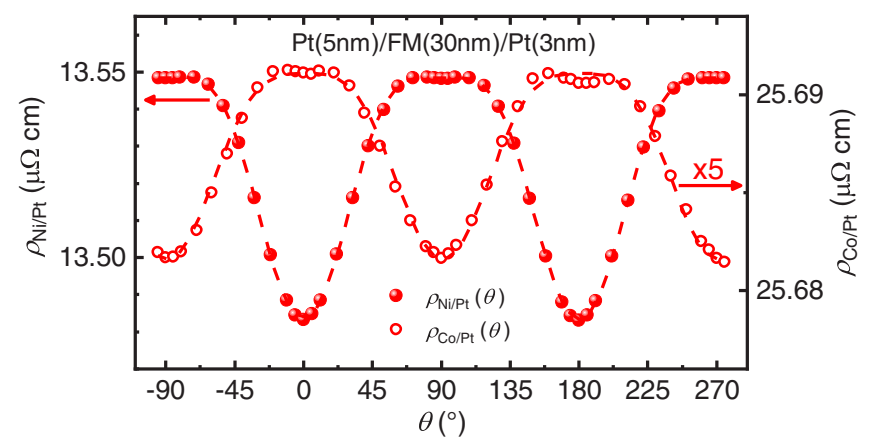

FIG. 3. Resistivity $\rho_{\mathrm{FM} / \mathrm{Pt}}$ vs angle $\theta$ for $\operatorname{Pt}(5 \mathrm{~nm}) / \mathrm{FM}(30 \mathrm{~nm}) /$ $\operatorname{Pt}(3 \mathrm{~nm})$ sandwiches, $\mathrm{FM}=(\mathrm{Co}, \mathrm{Ni})$. The dashed lines are fits to the data using a series expansion in $\cos ^{2 n} \theta$ up to $2 n=6$ [Eq. (4)]. For Ni (filled dots) and Co (open dots) the angular dependence is dominated by fourth-order and second-order contributions, respectively.

to be sensitive to higher orders we investigated the $\rho(\theta)$ behavior of (111)-textured $\mathrm{Pt} / \mathrm{Co} / \mathrm{Pt}$ sandwiches in the range where interface and bulk contributions of second order almost cancel (i.e., $t_{\mathrm{Co}} \approx 35 \mathrm{~nm}$ ) [31]. In fact, as can be seen from Fig. 3, where the results for $t_{\mathrm{Co}}=30 \mathrm{~nm}$ are shown (MR ratio of second order is $\Delta \rho_{\mathrm{op}}^{(2)} / \rho \approx 0.07 \%$ ), small dips surrounded by maxima around $\theta=\left(0^{\circ}, 180^{\circ}\right)$ are clearly observed reflecting the presence of a fourthorder contribution, which in turn supports the proposed generality of Eq. (5). The corresponding MR ratio is only $\Delta \rho_{\mathrm{op}}^{(4)} / \rho \approx-0.03 \%$ and therefore more than one order of magnitude smaller than for Pt/Ni/Pt with the same thickness of the ferromagnetic layer. The size of the sixth-order contribution, if present, is smaller than the experimental resolution of $10^{-5}$.

The symmetry reasoning and thus the same result of the derivation, i.e., Eq. (5), holds also for the interfacial AMR contributions as the abrupt interfaces give rise to a uniaxial symmetry as well. Hence, $\sin ^{2} \theta \cos ^{2} \varphi$ and $\cos ^{2 n} \theta$ terms can be expected as interfacial AMR contributions. Contributions due to Rashba-like spin-orbit scattering and SMR might be present as well. Thereby, the ratio of the amplitudes of the second-order contributions $B_{\text {ip, interface }} / B_{\text {op,interface }}=\Delta \rho_{\text {ip,interface }} / \Delta \rho_{\text {op,interface }}^{(2)}$ decides which of the effects dominate the MR behavior.

In order to properly check for the existence of interfacial MR contributions of $\mathrm{Pt} / \mathrm{Ni} / \mathrm{Pt}$ the modeling of the sandwich resistance by means of a combined parallel-current and Fuchs-Sondheimer model [71] following Ref. [30] has been performed (Supplemental Material III [67]). When doing so the pure MR amplitudes of the Ni layer, namely, the resistivity differences $\Delta \rho_{\mathrm{ip}, \mathrm{Ni}}$ and $\Delta \rho_{\mathrm{op}, \mathrm{Ni}}^{(2 n)}$ are obtained (effective single Ni layer model). The correction for the parasitic effect of shunting through the Pt layers finally enables a separation of bulk and interfacial MR effects via the dependence on Ni thickness. Note that a sample with 
$t_{\mathrm{Ni}}=1 \mathrm{~nm}$ sandwiched by $\mathrm{Pt}$ is not ferromagnetic at room temperature (checked by MOKE, Supplemental Material I [67]) due to the intermixing of $\mathrm{Ni}$ and $\mathrm{Pt}$ at the $\mathrm{Ni} / \mathrm{Pt}$ interfaces. The paramagnetic state is expected since $\mathrm{Ni}_{x} \mathrm{Pt}_{1-x}$ alloys are only ferromagnetic at room temperature for high $\mathrm{Ni}$ concentrations $(x>0.6)$ [80]. In order to correct for the magnetic dead layer (MDL) formation we rescaled the $\mathrm{Ni}$ thickness by $1 \mathrm{~nm}$, $t_{\mathrm{Ni} \text {,corr }}=t_{\mathrm{Ni}}-1 \mathrm{~nm}$, and the $\mathrm{NiPt}$ alloy is treated as additional paramagnetic material (Supplemental Material III [67]).

The resultant resistivity differences $\left(\Delta \rho_{\mathrm{ip}, \mathrm{Ni}}, \Delta \rho_{\mathrm{op}, \mathrm{Ni}}^{(2 n)}\right)$ vs $t_{\mathrm{Ni} \text {,corr }}$ are shown in Fig. 4 . The presence of interfacial MR contributions for $\Delta \rho_{\mathrm{op}, \mathrm{Ni}}^{(2)}$ can be recognized from the $\left(1 / t_{\mathrm{Ni}, \text { corr }}\right.$ )-like dependence (green dots). Quantitatively, the curve can be well described by the Fuchs-Sondheimer model [71] taking an anisotropy in the interfacial specularity parameter into account [30]: $\Delta p_{\text {op }}^{(2)}=(0.011 \pm 0.002)$. The small thickness-independent offset represents the crystalline bulk-AMR contribution due to the texture (GSE): $\Delta \rho_{\mathrm{op}, \mathrm{Ni} \text { bulk }}^{(2)}=-(0.004 \pm 0.007) \mu \Omega \mathrm{cm}$. In contrast to the second order, the resistivity difference of the fourth order appears to be almost constant (blue dots). The reason for the slight thickness-driven decrease of $\left|\Delta \rho_{\mathrm{op}, \mathrm{Ni}}^{(4)}\right|$ at large thicknesses $t_{\mathrm{Ni} \text {, corr }} \gtrsim 30 \mathrm{~nm}$ is not understood, yet. The modeling reveals $\Delta p_{\mathrm{op}}^{(4)}=-(0.003 \pm 0.003), \Delta \rho_{\mathrm{op}, \mathrm{Ni} \text { bulk }}^{(4)}=$ $-(0.072 \pm 0.018) \mu \Omega \mathrm{cm}$, which particularly confirms that the interface-scattering anisotropy of the fourth order is zero within the error margins [81]. Hence, the observed $\left(1 / t_{\mathrm{Ni}, \text { corr }}\right)$-like dependence of $\Delta \rho_{\mathrm{op}}^{(4)} / \rho$ [Fig. 2(b)] is a mere consequence of the above-mentioned decrease of $\left|\Delta \rho_{\mathrm{op}, \mathrm{Ni}}^{(4)}\right|$ at large $\mathrm{Ni}$ thicknesses. Importantly, contrary to $\mathrm{Pt} / \mathrm{Co} / \mathrm{Pt}$ [30], the resistivity difference for the conventional AMR geometry, $\Delta \rho_{\mathrm{ip}, \mathrm{Ni}}$, is basically constant (black dots), namely, the obtained fit parameter $\Delta p_{\text {ip }}$ is zero within the experimental resolution [81]: $\Delta p_{\text {ip }}=(0.001 \pm 0.002)$, $\Delta \rho_{\text {ip,Nibulk }}=(0.265 \pm 0.009) \mu \Omega \mathrm{cm}$. Small interfacial contribution, however, cannot be ruled out due to a possibly erroneous assumption of the thickness of the magnetic dead layers. In any case, the results lastingly prove that the interfacial contributions in $\Delta \rho_{\text {ip }}$ are much smaller than the ones in $\Delta \rho_{\mathrm{op}}^{(2)}$. Consequently, the equation for the SMR, i.e., $\Delta \rho_{\text {ip }}=\Delta \rho_{\text {op }}^{(2)}$ [Eq. (3)], does not describe the data. The result also violates the prediction for Rashba-based interfacial AMR, i.e., $1.5 \Delta \rho_{\text {ip,interface }}=\Delta \rho_{\text {op,interface }}^{(2)}$.

In conclusion, the AMR of fcc (111)-textured films is derived from the symmetry-adapted description for single crystals. The modeling reveals the conventional AMR-like angular dependence for in-plane rotation of magnetization $\left(\theta=90^{\circ}\right)$. Higher orders than $\cos ^{2} \varphi$ do not exist. In stark contrast to isotropic polycrystals and amorphous materials,

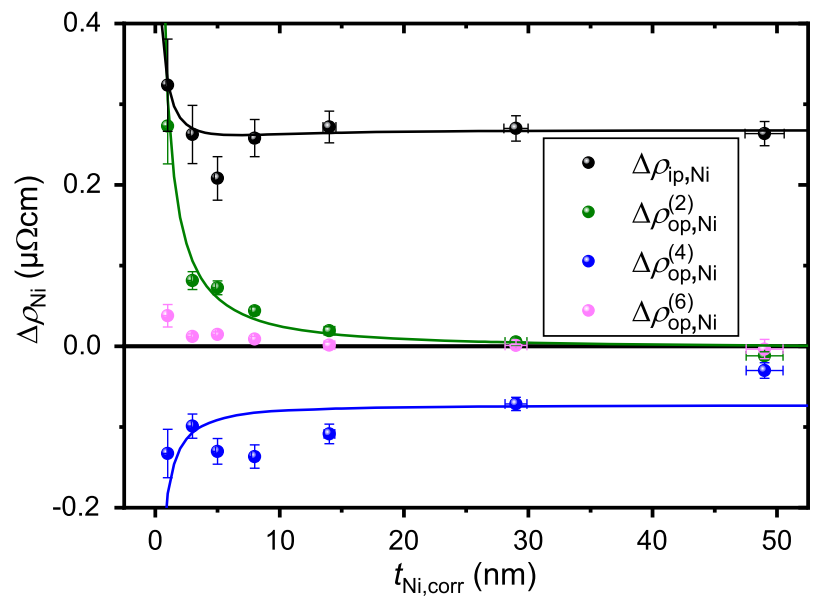

FIG. 4. Difference resistivity of the $\mathrm{Ni}$ layer $\Delta \rho_{\mathrm{ip}, \mathrm{Ni}}$ and $\Delta \rho_{\mathrm{op}, \mathrm{Ni}}^{(2 n)}, 2 n \leq 6$, as a function of $\mathrm{Ni}$ thickness corrected for MDL $t_{\mathrm{Ni}, \text { corr }}$. The solid lines are fits according to Eq. (S17) given in Supplemental Material III [67], particularly revealing the existence of interfacial contributions in $\Delta \rho_{\mathrm{op}, \mathrm{Ni}}^{(2)}$. The latter are absent in $\Delta \rho_{\mathrm{ip}, \mathrm{Ni}}$ and $\Delta \rho_{\mathrm{op}, \mathrm{Ni}}^{(4)}$ within the experimental resolution [81].

the modeling predicts $\cos ^{2 n} \theta$ dependences up to $2 n=4$ in polar rotational geometry $\left(\varphi=90^{\circ}\right)$ revealing that the assumption of an AMR in the conventional sense, namely, a constant resistivity in polar rotational geometry, is not valid for textured films due to symmetry reasons. It is therefore demonstrated that the GSE and the AIMR effects are not new physical effects but that they are simply a manifestation of the crystal symmetry and arrangement of grains, and the symmetry breaking at the interfaces, respectively. As a far-reaching consequence the appearance of $\cos ^{2} \theta$ terms in textured films cannot be used as a fingerprint for the presence of SMR in all-metallic systems. Even for amorphous films the interfaces can produce such AMR contributions. A distinction between SMR and AMR can only be made when properly quantifying the interfacial contributions. Here, for $\mathrm{Pt} / \mathrm{Ni} / \mathrm{Pt}$, the interfacial contributions in $\Delta \rho_{\text {ip }}$ are much smaller than the ones in $\Delta \rho_{\text {op }}^{(2)}$, demonstrating the negligible role of the SMR effect in allmetallic layered systems. In contrast to $\mathrm{Pt} / \mathrm{Co} / \mathrm{Pt}[30]$ the results also violate the prediction for Rashba-based interfacial AMR [47]. All in all, the MR behavior of $\mathrm{Pt} / \mathrm{Ni} / \mathrm{Pt}$ including higher order contributions in $\cos ^{2 n} \theta$ as well as $\Delta \rho_{\text {ip,interface }} \ll \Delta \rho_{\text {op,interface }}^{(2)}$ is only compatible with the symmetry-adapted modeling of the AMR of fcc (111)textured thin films.

Financial support by Deutsche Forschungsgemeinschaft (DFG) via Project No. OE 251/7-1 "Investigation of the influence of interfaces on the magnetotransport in ultrathin films" and Sonderforschungsbereich (collaborative research center) SFB 668 is gratefully acknowledged. 
*andre.kobs@desy.de

[1] W. Thomson, Proc. R. Soc. 8, 546 (1856).

[2] L. L. Campbell, Galvanomagnetic and Thermomagnetic Effects-The Hall and Allied Phenomena (Longmans, Green and Company, London, 1923).

[3] The phrase anisotropic magnetoresistance is widely accepted today. Note that before the 1970 s different synonyms for the AMR were used in literature, such as extraordinary $\mathrm{MR}$, ferromagnetic resistivity anisotropy, orientation effect, or spontaneous resistance anisotropy.

[4] J. Smit, Physica 17, 612 (1951).

[5] L. Berger, Physica 30, 1141 (1964).

[6] R. I. Potter, Phys. Rev. B 10, 4626 (1974).

[7] P. McCorkle, Phys. Rev. 22, 271 (1923).

[8] W. L. Webster, Proc. R. Soc. 113, 196 (1926).

[9] S. Kaya, Sci. Rep. Tohoku Imp. Univ. 17, 1027 (1928).

[10] R. Gans and J. v. Harlem, Ann. Phys. (Berlin) 407, 516 (1932).

[11] E. Englert, Ann. Phys. (Berlin) 406, 589 (1932).

[12] W. Döring, Ann. Phys. (Berlin) 424, 259 (1938).

[13] V. A. Marsocci, J. Appl. Phys. 35, 774 (1964).

[14] L. Berger and S. A. Friedberg, Phys. Rev. 165, 670 (1968).

[15] M. Tondra, D. Lottis, K. T. Riggs, Y. Chen, E. D. Dahlberg, and G. A. Prinz, J. Appl. Phys. 73, 6393 (1993).

[16] R. P. van Gorkom, J. Caro, T. M. Klapwijk, and S. Radelaar, Phys. Rev. B 63, 134432 (2001).

[17] A. W. Rushforth, K. Výborný, C. S. King, K. W. Edmonds, R. P. Campion, C. T. Foxon, J. Wunderlich, A. C. Irvine, P. Vašek, V. Novák, K. Olejník, Jairo Sinova, T. Jungwirth, and B. L. Gallagher, Phys. Rev. Lett. 99, 147207 (2007).

[18] Y. Bason, J. Hoffman, C. H. Ahn, and L. Klein, Phys. Rev. B 79, 092406 (2009).

[19] N. Naftalis, A. Kaplan, M. Schultz, C. A. F. Vaz, J. A. Moyer, C. H. Ahn, and L. Klein, Phys. Rev. B 84, 094441 (2011).

[20] X. Xiao, J. H. Liang, B. L. Chen, J. X. Li, D. H. Ma, Z. Ding, and Y.Z. Wu, J. Appl. Phys. 118, 043908 (2015).

[21] F. Takata, K. Kabara, M. Tsunoda, and T. Suemasu, J. Appl. Phys. 121, 023903 (2017).

[22] T. R. McGuire and R. I. Potter, IEEE Trans. Magn. 11, 1018 (1975).

[23] A. Kobs, S. Heße, H. P. Oepen, and P. Weinberger, Philos. Mag. 92, 2835 (2012).

[24] S. Kokado and M. Tsunoda, J. Phys. Soc. Jpn. 84, 094710 (2015).

[25] T. T. Chen and V. A. Marsocci, J. Appl. Phys. 43, 1554 (1972).

[26] S. N. Kaul, Acta Metall. 23, 1121 (1975).

[27] T. G. S. M. Rijks, S. K. J. Lenczowski, R. Coehoorn, and W. J. M. de Jonge, Phys. Rev. B 56, 362 (1997).

[28] M. Brands and G. Dumpich, J. Appl. Phys. 98, 014309 (2005).

[29] W. Gil, D. Görlitz, M. Horisberger, and J. Kötzler, Phys. Rev. B 72, 134401 (2005).

[30] A. Kobs and H.P. Oepen, Phys. Rev. B 93, 014426 (2016).

[31] A. Kobs, S. Heße, W. Kreuzpaintner, G. Winkler, D. Lott, P. Weinberger, A. Schreyer, and H. P. Oepen, Phys. Rev. Lett. 106, 217207 (2011).
[32] J.-C. Lee, C.-H. Hsieh, C.-C. Chang, L.-W. Huang, L.-K. Lin, and S.-F. Lee, J. Appl. Phys. 113, 17C714 (2013).

[33] M. Hille, A. Frauen, B. Beyersdorff, A. Kobs, S. Heße, R. Frömter, and H. P. Oepen, J. Appl. Phys. 113, 023902 (2013).

[34] Y. M. Lu, J. W. Cai, S. Y. Huang, D. Qu, B. F. Miao, and C. L. Chien, Phys. Rev. B 87, 220409(R) (2013); A. Kobs, A. Frauen, and H. P. Oepen, Phys. Rev. B 90, 016401 (2014).

[35] Y. Kachlon, N. Kurzweil, and A. Sharoni, J. Appl. Phys. 115, 173911 (2014).

[36] J. X. Li, M. W. Jia, Z. Ding, J. H. Liang, Y. M. Luo, and Y. Z. Wu, Phys. Rev. B 90, 214415 (2014).

[37] C. O. Avci, K. Garello, J. Mendil, A. Ghosh, N. Blasakis, M. Gabureac, M. Trassin, M. Fiebig, and P. Gambardella, Appl. Phys. Lett. 107, 192405 (2015).

[38] A. Frauen, A. Kobs, T. Böhnert, A.-K. Michel, G. Winkler, K. Nielsch, and H. P. Oepen, Phys. Rev. B 92, 140402(R) (2015).

[39] J. Liu, T. Ohkubo, S. Mitani, K. Hono, and M. Hayashi, Appl. Phys. Lett. 107, 232408 (2015).

[40] S. Cho, S.-h. Chris Baek, K.-D. Lee, Y. Jo, and B.-G. Park, Sci. Rep. 5, 14668 (2015).

[41] K. Meng, J. Xiao, Y. Wu, J. Miao, X. Xu, J. Zhao, and Y. Jiang, Sci. Rep. 6, 20522 (2016).

[42] Y. Zhang, H. Fu, N. Sun, W. Che, D. Ding, J. Qin, C. You, R. Shan, and Z. Zhu, J. Magn. Magn. Mater. 411, 103 (2016).

[43] Q. Liu, G. Yang, Y. W. Liu, Y. Cao, J. Y. Zhang, M. H. Li, L. Ding, D. W. Wang, Q. Zhan, C. Feng, Y. D. Pan, and G. H. Yu, IEEE Magn. Lett. 8, 1 (2017).

[44] Y. Yang, Z. Luo, H. Wu, Y. Xu, R.-W. Li, S. J. Pennycook, S. Zhang, and Y. Wu, Nat. Commun. 9, 2255 (2018).

[45] L. K. Zou, Y. Zhang, L. Gu, J. W. Cai, and L. Sun, Phys. Rev. B 93, 075309 (2016).

[46] M. Harder, Y. Gui, and C.-M. Hu, Phys. Rep. 661, 1 (2016).

[47] S. S.-L. Zhang and S. Zhang, J. Appl. Phys. 115, 17 C703 (2014).

[48] J. E. Hirsch, Phys. Rev. Lett. 83, 1834 (1999).

[49] J. Sinova, S. O. Valenzuela, J. Wunderlich, C. H. Back, and T. Jungwirth, Rev. Mod. Phys. 87, 1213 (2015).

[50] I. M. Miron, K. Garello, G. Gaudin, P.-J. Zermatten, M. V. Costache, S. Auffret, S. Bandiera, B. Rodmacq, A. Schuhl, and P. Gambardella, Nature (London) 476, 189 (2011).

[51] L. Q. Liu, C.-F. Pai, Y. Li, H. W. Tseng, D. C. Ralph, and R. A. Buhrman, Science 336, 555 (2012).

[52] F. Hellman et al., Rev. Mod. Phys. 89, 025006 (2017).

[53] H. Nakayama, M. Althammer, Y.-T. Chen, K. Uchida, Y. Kajiwara, D. Kikuchi, T. Ohtani, S. Geprägs, M. Opel, S. Takahashi, R. Gross, G. E. W. Bauer, S. T. B. Goennenwein, and E. Saitoh, Phys. Rev. Lett. 110, 206601 (2013).

[54] Y.-T. Chen, S. Takahashi, H. Nakayama, M. Althammer, S. T. B. Goennenwein, E. Saitoh, and G. E. W. Bauer, J. Phys. Condens. Matter 28, 103004 (2016).

[55] J. Kim, P. Sheng, S. Takahashi, S. Mitani, and M. Hayashi, Phys. Rev. Lett. 116, 097201 (2016).

[56] T. Okuno, T. Taniguchi, S. Kim, S.-h- Chris Baek, B.-G. Park, T. Moriyama, K.-J. Kim, and T. Ono, Jpn. J. Appl. Phys. 55, 080308(R) (2016). 
[57] J.-G. Choi, J. W. Lee, and B.-G. Park, Phys. Rev. B 96, 174412 (2017).

[58] Y.-C. Lau and M. Hayashi, Jpn. J. Appl. Phys. 56, 0802B5 (2017).

[59] M. Kawaguchi, D. Towa, Y.-C. Lau, S. Takahashi, and M. Hayashi, Appl. Phys. Lett. 112, 202405 (2018).

[60] X. Xiao, J. X. Li, Z. Ding, J. H. Liang, L. Sun, and Y. Z. Wu, Appl. Phys. Lett. 108, 222402 (2016).

[61] S.-Y. Huang, H.-L. Li, C.-W. Chong, Y.-Y. Chang, M.-K. Lee, and J.-C.-A. Jung, Sci. Rep. 8, 108 (2018).

[62] F. S. M. Guimarães, M. dos Santos Dias, J. Bouaziz, A. T. Costa, R. B. Muniz, and S. Lounis, Sci. Rep. 7, 3686 (2017).

[63] The same criticisms apply for studies on Rashba-Edelstein magnetoresistance, see, e.g., Refs. [64,65].

[64] H. Nakayama, Y. Kanno, H. An, T. Tashiro, S. Haku, A. Nomura, and K. Ando, Phys. Rev. Lett. 117, 116602 (2016).

[65] J. Kim, Y.-T. Chen, S. Karube, S. Takahashi, K. Kondou, G. Tatara, and Y. C. Otani, Phys. Rev. B 96, 140409(R) (2017).

[66] G. Winkler, A. Kobs, A. Chuvilin, D. Lott, A. Schreyer, and H. P. Oepen, J. Appl. Phys. 117, 105306 (2015).

[67] See Supplemental Material at http://link.aps.org/ supplemental/10.1103/PhysRevLett.123.137201 for (I) structural and magnetic characterization, which includes Refs. [68,31,69,70], (II) for derivation of AMR for fcc (111) textured films which includes Refs. [12,22,29], and (III) for modeling of electrical transport for $\mathrm{Pt} / \mathrm{Ni} / \mathrm{Pt}$ sandwiches, which includes Refs. [30,71,72].

[68] A. Nelson, J. Appl. Crystallogr. 39, 273 (2006).

[69] B. Raquet, M. Viret, E. Sondergard, O. Cespedes, and R. Mamy, Phys. Rev. B 66, 024433 (2002).

[70] J. Zak, E. R. Moog, C. Liu, and S. D. Bader, J. Magn. Magn. Mater. 89, 107 (1990).
[71] E. H. Sondheimer, Adv. Phys. 1, 1 (1952); 50, 499 (2001).

[72] J. M. Lavine, Phys. Rev. 123, 1273 (1961).

[73] Investigations of the temperature dependence of the MR indicate significant sixth-order contributions at low temperatures $2 \mathrm{~K} \leq T \leq 200 \mathrm{~K}$ (not shown).

[74] Y.-T. Chen, S. Takahashi, H. Nakayama, M. Althammer, S. T. B. Goennenwein, E. Saitoh, and G. E. W. Bauer, Phys. Rev. B 87, 144411 (2013).

[75] X. Xiao, X. J. X. Li, Z. Ding, and Y.Z. Wu, J. Appl. Phys. 118, 203905 (2015).

[76] A. C. Smith, J.F. Janak, and R. B. Adler, Electronic Conduction in Solids (McGraw-Hill, New York, 1967).

[77] The expression for the AMR for various crystal lattices can be extracted from Ref. [78].

[78] W. Döring and G. Simon, Ann. Phys. (N.Y.) 460, 373 (1960).

[79] Note that higher orders in $\cos ^{2 n} \theta, 2 n \geq 6$ are missing in the modeling [Eq. (5)] due to the early termination of the series expansion [Eq. (S3), Supplemental, II [67]].

[80] T. Golod, A. Rydh, and V. M. Krasnov, J. Appl. Phys. 110, 033909 (2011).

[81] Note that for a bulklike AMR $\Delta \rho_{\text {ip,Nibulk }} \neq 0\left(\Delta \rho_{\text {op,Ni bulk }}^{(4)} \neq 0\right)$ and a vanishing anisotropy of the interface scattering $\Delta p_{\text {ip }}=0\left(\Delta p_{\mathrm{op}}^{(4)}=0\right)$ a steep decrease of $\Delta \rho_{\mathrm{ip}, \mathrm{Ni}}\left(t_{\mathrm{Ni}, \mathrm{corr}}\right)$ $\left(\Delta \rho_{\mathrm{op}, \mathrm{Ni}}^{(4)}\left(t_{\mathrm{Ni}, \mathrm{corr}}\right)\right)$ occurs in the limit where the film thickness is smaller than the bulk mean free path (for specularity parameters $p<1)[30,82,83]$.

[82] T. G. S. M. Rijks, R. Coehoorn, M. J. M. de Jong, and W. J. M. de Jonge, Phys. Rev. B 51, 283 (1995).

[83] B. Dieny, M. Li, S. H. Liao, C. Horng, and K. Ju, J. Appl. Phys. 88, 4140 (2000). 